\title{
Funções Executivas: Relação entre Relatos de Pais, de Professores e Desempenho de Crianças
}

\author{
Alana Tosta Martoni \\ Educação Infantil e Ensino Fundamental I da Prefeitura Municipal de São Paulo, \\ São Paulo, SP, Brasil \\ Bruna Tonietti Trevisan \\ Programa de Pós-Graduação em Distúrbios do Desenvolvimento da Universidade \\ Presbiteriana Mackenzie, São Paulo, SP, Brasil \\ Natália Martins Dias ${ }^{1}$ \\ Programa de Pós-Graduação em Psicologia Educacional do Centro Universitário Fundação \\ Instituto de Ensino para Osasco - FIEO, Osasco, SP, Brasil \\ Alessandra Gotuzo Seabra \\ Programa de Pós-Graduação em Distúrbios do Desenvolvimento da Universidade \\ Presbiteriana Mackenzie, São Paulo, SP, Brasil
}

\section{Resumo}

Funções executivas (FE) possibilitam ao indivíduo regular seu comportamento. Em crianças, têm sido avaliadas por meio de testes de desempenho e escalas preenchidas por pais/professores. Porém, a literatura tem demonstrado que a concordância entre diferentes tipos de medidas psicológicas e entre diferentes respondentes é, no máximo, moderada. Este estudo investigou: (a) a concordância entre pais e professores em escalas de avaliação de FE e de indicadores de desatenção e hiperatividade, e (b) as relações entre as pontuações nas escalas e o desempenho de crianças em um teste de inibição, um componente das FE. Participaram 144 crianças (idade média $=6,16 a$ ) de $1^{\mathrm{a}}$ e $2^{\mathrm{a}}$ fase do Ensino Infantil e $1^{\circ}$ ano do Fundamental de uma escola pública de uma cidade da Grande São Paulo, seus pais e professores. Pais e professores responderam ao Inventário de Funcionamento Executivo Infantil (IFEI) e ao MTA-SNAP-IV. As crianças responderam ao Teste de Stroop Semântico. Houve correlações significativas, baixas a moderadas, entre respostas de pais e de professores nas escalas, sendo essas correlações mais frequentes nas crianças da $1^{a}$ fase. Pais indicaram mais dificuldades nas crianças do que os professores. Foram encontradas correlações, de baixas a moderadas, entre desempenho das crianças no Stroop e respostas dos informantes. Com a progressão escolar, o IFEI tendeu a se correlacionar com medidas mais complexas do Stroop. Portanto, houve concordância, em geral baixa, entre escalas e teste de desempenho, assim como entre respostas de pais e de professores, corroborando a importância de considerar diferentes fontes de informação na avaliação infantil.

Palavras-chave: Processos cognitivos, desenvolvimento infantil, avaliação infantil, funções executivas, atenção.

Endereço para correspondência: UniFIEO - Centro Universitário Fundação Instituto de Ensino para Osasco, Programa de Pós Graduação Stricto Sensu - Psicologia Educacional, Avenida Franz Voegeli, 300, Bloco Prata, Bairro Continental, Osasco, SP, Brasil 06020-190. Fone: (11) 3651-9914. E-mail: alana.martoni@gmail.com, brunattrevisan@gmail.com, natalia.dias@unifieo.br e alessandragseabra@gmail.com

Apoio financeiro: Fundação de Amparo à Pesquisa do Estado de São Paulo (FAPESP) e Conselho Nacional de Desenvolvimento Científico e Tecnológico (CNPq). 


\title{
Executive Functions: Relation between Evaluation by Parents and Teachers and the Performance of Children
}

\begin{abstract}
Executive functions (EF) enable us to regulate our behavior. In children, these abilities have been evaluated by performance tests and scales filled by parents/teachers. However, the literature has shown that the correlation between different types of psychological measurements and between different respondents is, at best, moderate. This study investigated: (a) the agreement between parents and teachers in rating scales of EF and indicators of inattention and hyperactivity, and (b) the relationship between scores on the scales and the performance of children on a test of inhibition, a component of EF. Participants were 144 children (mean age $=6.16 \mathrm{y}$ ) from $1^{\text {st }}$ and $2^{\text {nd }}$ phases of Children's Education and $1^{\text {st }}$ grade of Elementary of a public school in a town of Sao Paulo, their parents and teachers. Parents and teachers completed the Childhood Executive Functioning Inventory (CHEXI) and MTA-SNAP-IV. Children responded to the Semantic Stroop Test. There were significant correlations, low to moderate, between responses of parents and teachers on the scales. Correlations were more frequent in $1^{\text {st }}$ phase children. Parents indicated more difficulties in children than teachers did. Low to moderate correlations between children's performance on the Stroop and responses of informants were found. With school progression, the CHEXI tended to correlate with increasingly complex measures of Stroop. Therefore, there was concordance, low in general, between scales and performance testing, as well as between responses of parents and teachers, corroborating the importance of considering different sources of information on child assessment.
\end{abstract}

Keywords: Cognitive process, child development, child assessment, executive functions, attention.

\section{Funciones Ejecutivas: Relación entre Evaluación por Padres, Maestros y Rendimiento de los Niños}

\section{Resumen}

Funciones ejecutivas (FE) permiten regular lo comportamiento. En niños, han sido evaluados por pruebas de rendimiento y escalas respondidas por padres/maestros. La literatura ha demostrado que la correlación entre diferentes tipos de medidas e entre diferentes informantes es, el mejor, moderado. Este estudio investigó: (a) el acuerdo entre padres y maestros en escalas de evaluación de FE e de indicadores de desatención e hiperactividad, y (b) la relación entre puntuaciones en escalas y rendimiento de los niños en una prueba de inhibición, un componente de FE. Participaron 144 niños (edad media=6,16a) de las $1^{\mathrm{a}}$ e $2^{\mathrm{a}}$ fases de la Educación Infantil y de lo $1^{\mathrm{o}}$ año de la Primaria de una escuela pública de São Paulo, sus padres y maestros. Padres y maestros completaron el Inventario de funcionamiento ejecutivo Infantil (IFEI) y MTA-SNAP-IV. Los niños respondieron a la prueba de Stroop Semántico. Hubo correlaciones significativas, bajas a moderadas, entre las respuestas de padres y maestros en las escalas, siendo estas más comunes en la $1^{a}$ fase. Los padres indicaron más dificultades en los niños que los maestros. Fueron encontradas correlaciones, bajas a moderadas, entre el rendimiento de los niños en el Stroop y las respuestas de los informantes. Con la progresión escolar, el IFEI tendió a correlacionarse con medidas más complejas de Stroop. Por tanto, hubo concordancia, en general baja, entre escalas y pruebas de rendimiento, así como entre las respuestas de padres y maestros, lo que corrobora la importancia de considerar diferentes fuentes de información in la evaluación infantil.

Palabras clave: Procesos cognitivos, desarrollo infantil, evaluación infantil, funciones ejecutivas, atención. 
Funções executivas são habilidades que possibilitam ao indivíduo controlar e regular o processamento da informação e seu comportamento (Diamond, 2013; Gazzaniga, Ivry, \& Mangun, 2006). Estas habilidades são requeridas sempre que, devido à novidade ou à complexidade da tarefa ou situação, o processamento automático não é adequado ou é insuficiente para solucioná-la (Menezes, Godoy, Teixeira, Carreiro, \& Seabra, 2012). Estas habilidades têm sido bastante abordadas na literatura, sobretudo no que tange à multidimensionalidade do construto (Fonseca et al., 2010). Há relativo consenso de que as funções executivas consistem de três habilidades principais, a inibição, a memória de trabalho e a flexibilidade cognitiva (Diamond, 2013; Miyake, Friedman, Emerson, Witzki, \& Howerter, 2000). Outras habilidades frequentemente mencionadas na literatura como associadas às funções executivas, como o planejamento e a tomada de decisões (Gazzaniga et al., 2006; Lezak, Howieson, \& Loring, 2004; Malloy-Diniz, Sedo, Fuentes, \& Leite, 2008), são consideradas habilidades mais complexas e que resultariam da integração das três habilidades principais.

A inibição tem duas funções principais. Uma é relacionada à inibição de resposta, por exemplo, ao inibir um comportamento em um ambiente em que ele não é adequado, permitindo ao indivíduo controlar sua ação e responder apropriadamente às exigências do ambiente, ou seja, exercer autocontrole. A outra função relaciona-se à inibição cognitiva, que se refere à inibição de pensamentos e de distratores (Diamond, 2013; Nigg, 2001), estando intrinsecamente relacionada à atenção seletiva, por sua vez compreendida como um mecanismo que possibilita processar informações relevantes, enquanto ignora outras irrelevantes ou dispersivas (Gazzaniga et al., 2006). A memória de trabalho refere-se à capacidade de sustentar a informação mentalmente para utilizá-la em uma dada tarefa, integrá-la a outras informações ou enquanto executa outra atividade. Ela permite que a informação seja manipulada, ou seja, que o indivíduo possa operar com e transformar a informação (Baddeley, 2000; Diamond, 2013; Gazzaniga et al., 2006). A flexibilidade cognitiva pode ser considerada mais complexa que as duas primeiras visto que depende, em alguma medida, das habilidades anteriores. A flexibilidade permite ao indivíduo lidar com diversas informações e tarefas simultaneamente. Está relacionada à habilidade de mudar de perspectiva e é fundamental para a capacidade de adaptar o comportamento às mudanças nas situações e demandas sociais (Diamond, 2013).

O desenvolvimento de tais habilidades começa a ocorrer por volta do primeiro ano de vida. Considera-se que a primeira habilidade a emergir, em torno dos 12 meses, seja o controle inibitório, sendo que memória de trabalho e flexibilidade iniciam seu desenvolvimento após essa idade. Apesar deste início precoce, o desenvolvimento das funções executivas perdura por toda infância, até meados da adolescência e vida adulta inicial. Na faixa etária pré-escolar, foco deste estudo, há um importante desenvolvimento destas habilidades. Entre quatro e cinco anos as crianças tornam-se progressivamente mais hábeis em focalizar a atenção, controlar o comportamento e ter maior controle de suas emoções, sendo mais capazes de seguir regras e adequar seu comportamento às demandas sociais. Entre cinco e seis anos, são capazes de planejar e solucionar problemas simples (Dawson \& Guare, 2010; García-Molina, Enseñat-Cantallops, Tirapu-Ustárroz, \& Roig-Rovira, 2009). Uma revisão acerca do desenvolvimento das funções executivas pode ser consultada em Dias e Seabra (2013).

No ambiente escolar, tais habilidades são requisitadas e podem ser fomentadas, visto que as situações apresentadas demandam da criança soluções para os problemas propostos, planejamento e adaptação a situações variadas na busca de novos objetivos, características essas intrinsicamente relacionadas às funções executivas. Assim, se por um lado o ambiente escolar, por exigir tais habilidades, pode ter potencial para fomentá-las, por outro, é fato que crianças que ingressam na escola com pobres funções executivas são mais propensas a apresentar dificuldades no controle de seu comportamento, na 
aprendizagem e na organização para trabalhos e atividades. Tais dificuldades tendem a aumentar com a complexidade crescente das tarefas no curso dos anos escolares, que impõem demandas crescentes às suas, já pobres, habilidades executivas (Dawson \& Guare, 2010).

De fato, o desempenho escolar, medido em termos de notas, está positivamente relacionado às funções executivas (Capovilla \& Dias, 2008). Há ainda indícios de que crianças que começam a escolarização com essas funções mais rebaixadas tendem a se tornar cada vez mais resistentes à escolarização, a esforçar-se cada vez menos e abandonar mais rapidamente as atividades (Blair \& Razza, 2007; Vitaro, Brendgen, Larose, \& Tremblay, 2005). Adicionalmente, segundo Blair e Diamond (2008), a dificuldade das crianças em atender às demandas escolares pode produzir nos professores atitudes de raiva $\mathrm{e}$ frustração, ajudando a fomentar na criança sentimentos de fracasso e afastamento do aluno do ambiente em questão. Em contrapartida, crianças com níveis mais elevados de funções executivas tendem a gostar de receber instrução e dedicar-se mais às tarefas, considerando a escola fácil e com atividades prazerosas (Vitaro et al., 2005).

Alterações nas funções executivas também têm se mostrado relacionadas a transtornos cognitivos e neuropsiquiátricos, tais como o Transtorno do Déficit de Atenção e Hiperatividade TDAH (Gonçalves et al., 2013; Willcutt, Doyle, Nigg, Faraone, \& Pennington, 2005), no qual, segundo Barkley (1997), há um rebaixamento especialmente de controle inibitório. Ainda, relações significativas, apesar de baixas, entre habilidades das funções executivas e indicadores de desatenção e hiperatividade foram encontradas em amostra não-clínica formada por crianças pré-escolares saudáveis em um estudo realizado previamente (Pereira, León, Dias, \& Seabra, 2012). Os sinais de TDAH já mencionados, a saber, desatenção e hiperatividade, podem ocasionar diferentes comprometimentos, por exemplo, interações inapropriadas com os pais, levando a conflitos também no ambiente familiar (Guilherme, Mattos, Serra-Pinheiro, \& Regalla, 2007). Especificamente em relação ao desempenho acadêmico, indivíduos com TDAH tendem a apresentar maior dificuldade em concluir os estudos, com mais chances de repetência e rendimento baixo quando comparado com seus pares (Malloy-Diniz et al., 2008). Em conjunto, tais evidências revelam a importância de se estudar o desenvolvimento das funções executivas e sua relação com sinais de desatenção e hiperatividade em indivíduos no ambiente escolar.

Dessa forma, tanto o déficit executivo em si mesmo, quanto os sinais de desatenção e hiperatividade que podem estar presentes em crianças podem influenciar o seu funcionamento escolar e familiar. Logo, é fundamental que se avalie precocemente as habilidades executivas e sinais de desatenção e de hiperatividade em crianças já a partir da idade pré-escolar, pois a identificação de alterações pode permitir a introdução de intervenções em fases precoces (Pereira et al., 2012).

Há diferentes formas de avaliação, tais como testes de desempenho e medidas funcionais, como questionários e escalas, que tendem a ser bastante utilizados para rastreio de determinados déficits ou transtornos. Existe uma tendência em se esperar resultados uniformes ao aplicar diferentes medidas (por exemplo, teste de desempenho $\mathrm{x}$ escala) que avaliem o mesmo construto ou construtos relacionados, ou quando se utiliza o mesmo instrumento de rastreio para diferentes informantes (como pai x professor), quando se intenciona, por exemplo, obter informações sobre o comportamento da criança. Contudo, nem sempre os resultados evidenciam uma homogeneidade (Major \& Seabra-Santos, 2013; Pereira et al., 2012; Seabra-Santos \& Gaspar, 2012).

Um estudo relativamente recente que investigou essa questão em crianças pré-escolares é o de Seabra-Santos e Gaspar (2012). Os autores avaliaram diferentes aspectos do desenvolvimento infantil a partir de uma escala respondida por pais e professores. O grau de acordo entre pais e professores variou de baixo a moderado e os pais apresentaram uma tendência a avaliar as crianças com melhores desempenhos em comparação aos professores. Os autores também verificaram a correlação entre informes de pais e professores e o desempenho das crianças em 
um teste de QI. As classificações de pais de professores se relacionaram de modo apenas baixo, no máximo moderado, com o desempenho das crianças no teste de QI e não foram notadas grandes discrepâncias nas correlações estabelecidas entre o desempenho no teste e os relatos de pais ou de professores. Os autores concluíram que os diferentes ambientes (casa x escola) permitem diferentes possibilidades de observação dos comportamentos. Também em amostra de pré-escolares, outro estudo revelou que a avaliação de indicadores de desatenção e hiperatividade pelo professor apresentou maior número de correlações, apesar de baixas, com medidas em testes de desempenho quando comparada à mesma avaliação realizada pelos pais (Pereira et al., 2012).

As divergências entre respostas por diferentes informantes têm recebido maior atenção da literatura e sido foco de discussão por pesquisadores. Tais discrepâncias entre relatos podem ser devidas, ao menos em parte, às expectativas em relação à criança e ao seu comportamento em distintos ambientes (Homer et al., 2000). A avaliação com recurso a escalas é, em algum grau, subjetiva e as divergências observadas nos diversos estudos podem refletir diferentes julgamentos e interpretações dos itens do instrumento, assim como sofrer influência de diversos outros fatores, entre elas a relação que o adulto tem com a criança ou adolescente avaliado (Zucker, Morris, Ingram, Morris, \& Bakeman, 2002). Segundo Major e Seabra-Santos (2013), as escalas de avaliação de comportamento têm assumido papel importante, sobretudo na avaliação de crianças na faixa etária pré-escolar, possibilitando a coleta de informações sobre distintos aspectos do comportamento em diferentes situações e contextos. O grau de concordância entre diferentes informantes, porém, é apenas modesto e diversos fatores podem estar relacionados a isso, entre eles a tendência de um informante ser muito benevolente/crítico, falta de compreensão dos itens do instrumento ou desconhecimento do comportamento (espectro normal-patológico) avaliado, além de erros inerentes aos próprios itens, que podem por exemplo referir-se a situa- ções com significados distintos para os diferentes informantes, sendo interpretados diferentemente por cada um deles. Além, segundo os mesmos autores, o contexto de avaliação, ou seja, a situação ambiental (estímulos, reforçadores) em que o comportamento pode ou não ocorrer, e a subjetividade do informante podem ser fatores intrincados na forma como pais e professores preenchem escalas de avaliação do comportamento infantil.

Especificamente com relação ao TDAH, estudos também têm encontrado divergências entre relatos de pais e professores. Ao investigar a concordância de pais e professores com relação aos sintomas de crianças e adolescentes com o transtorno, Mitsis, McKay, Schulz, Newcorn e Halperin (2000) evidenciaram a baixa relação entre respostas, principalmente referente aos subtipos do transtorno. O mesmo tem sido observado em estudos nacionais. Assim, em amostra clínica de crianças e adolescentes, idades entre seis e 16 anos e com recurso à MTA-SNAP-IV, pesquisadores verificaram que os professores são mais conservadores no relato de sintomas do que os pais. Estes últimos apresentaram maior queixa sobre o comportamento dos filhos, relatando altas taxas de sintomas em comparação com os professores (Coutinho, Mattos, Schmitz, Fortes, \& Borges, 2009). Com o mesmo instrumento de rastreio, porém em amostra não-clínica de crianças e adolescentes, idades entre 10 e 16 anos, outro estudo proveu evidências de que pais e professores têm diferentes perfis de pontuação para as subescalas da MTA-SNAP-IV e são provavelmente influenciados por aspectos culturais (Serra-Pinheiro, Mattos, \& Regalla, 2008).

Diante da necessidade de avaliar precocemente as funções executivas e possíveis sinais de desatenção e hiperatividade em crianças, bem como diante da divergência, exposta na literatura, entre relatos de diferentes informantes, o presente estudo avaliou crianças pré-escolares e no início do Ensino Fundamental em um teste de funções executivas, especificamente de atenção seletiva e controle inibitório, e em escalas de funcionamento executivo e de sinais de desatenção e hiperatividade, respondidas por pais 
e professores. Os objetivos desta investigação envolvem: (a) verificar a relação entre as escalas preenchidas por pais e por professores, e (b) investigar as relações entre as pontuações nas escalas e o desempenho das crianças no teste de desempenho. A hipótese do estudo é de que haverá concordância, porém baixa, entre respostas de pais e professores às escalas utilizadas. De forma análoga, a concordância entre as diferentes medidas (escala $\mathrm{x}$ teste de desempenho) deve ser baixa, possivelmente moderada entre medidas específicas (ex: subescala de controle inibitório da escala de avaliação das funções executivas e teste de desempenho, que avalia inibição).

\section{Método}

\section{Participantes}

Participaram crianças em fase pré-escolar e escolar, com idades entre 4 e 7 anos, e seus respectivos pais e professores. A amostra foi de conveniência, tendo participado da pesquisa todos os alunos das classes de Educação Infantil e do $1^{\circ}$ ano do Ensino Fundamental I cujos pais assim autorizaram. Os participantes foram 144 alunos, de ambos os sexos, de uma única Escola Municipal de Educação Infantil e Ensino Fundamental I, localizada em um bairro com nível socioeconômico médio e médio-baixo de uma cidade da grande São Paulo. A amostra foi composta por 38 alunos da $1^{\text {a }}$ fase da Educação Infantil (idade média $=5,1$ anos; $D P=0,29$ ), 33 da $2^{\mathrm{a}}$ fase da Educação Infantil (idade média = $6,3$ anos; $D P=0,29)$ e 73 crianças do $1^{\circ}$ ano do Ensino Fundamental I (idade média $=6,43$ anos; $D P=0,35)$.

\section{Instrumentos}

MTA-SNAP-IV. O MTA-SNAP-IV (Swanson, Nolan, \& Pelham - IV Questionnaire, ou SNAP-IV, versão utilizada no 'Multimodal Treatment Study for ADHD' - MTA) inclui 26 itens relativos aos sintomas do critério A do DSM-IV para TDAH (subescalas de desatenção e hiperatividade/impulsividade, cada uma com nove itens) e aos sintomas de Transtorno Desafiador e de Oposição. Neste estudo foram apenas utilizadas as subescalas de desatenção (ex: "Parece não estar ouvindo quando se fala diretamente com ele") e hiperatividade/impulsividade ("Sai do lugar na sala de aula ou em outras situações em que se espera que fique sentado"). O instrumento foi adaptado e validado no Brasil por Mattos, Serra-Pinheiro, Rohde e Pinto (2006). É respondido por pais e professores que pontuam, em uma escala Likert de quatro níveis, o quanto cada afirmativa é característica do comportamento da criança. Maior pontuação em cada subescala indica maior presença de indicadores do transtorno.

Inventário de Funcionamento Executivo Infantil (IFEI). A versão brasileira (Trevisan et al., 2011; Trevisan, Dias, Berberian, \& Seabra, no prelo) foi traduzida e adaptada do questionário de Thorell e Nyberg (2008), o Childhood Executive Functioning Inventory. O IFEI é composto por 26 itens, divididos em quatro subescalas: memória de trabalho (ex: "Tem dificuldade em entender instruções verbais a menos que também seja mostrado como fazer algo"), planejamento ("Tem dificuldade com tarefas ou atividades que envolvam várias etapas"), controle inibitório ("Tem tendência para fazer coisas sem pensar primeiro no que poderia acontecer") e regulação ("Tem clara dificuldade em fazer coisas que considera chatas"). É preenchido por pais e professores, que respondem, em uma escala Likert de cinco pontos, o quanto cada afirmativa é característica do comportamento da criança. Maiores pontuações indicam maior dificuldade.

Teste de Stroop Semântico (TSS). Avalia o componente de inibição das FE, incluindo atenção seletiva e controle inibitório. Nesta versão, desenvolvida com base em Berwid, Kera, Santra, Bender e Halperin (2005), as palavras, estímulos originais do teste de Stroop, são substituídas por pares de figuras correspondentes a "dia" e "noite", "menino" e "menina", e "grande" e "pequeno". Na primeira parte do teste, a criança deve nomear as figuras e, na segunda, deve dizer o substantivo oposto (exemplo: dizer "menino" diante da figura de "menina"). Cada parte do teste possui 16 itens totalizando 32 figuras, que são apresentadas em um tempo de 1200 milésimos 
de segundo para a primeira parte e 800 milésimos para a segunda parte. $\mathrm{Na}$ execução do teste foram registrados acertos e tempos de reação para a primeira e segunda partes do teste. Além, foram calculados o acerto e o tempo de reação de interferência. Ou seja, média de acertos na parte 2 (incongruente) subtraindo a média de acertos na parte 1 (congruente), e o tempo de reação médio da parte 2 (incongruente) subtraindo o tempo de reação médio da parte 1 (congruente). Evidências de validade do TSS em crianças brasileiras estão publicadas em Trevisan (2010).

\section{Procedimento}

Após a aprovação do Comitê de Ética, foi realizado contato com a Secretaria de Educação do município e, por intermédio desta, com a direção da escola participante. Após o consentimento da diretora da escola, os pais foram contatados em ocasião da reunião de pais e mestres, quando foram explicados os objetivos e procedimentos do estudo e entregues os Termos de Consentimento Livre e Esclarecido. Após aproximadamente uma semana, com a devolução dos termos assinados, iniciou-se a coleta dos dados. As crianças foram avaliadas, individualmente, no TSS em uma sessão única com duração aproximada de 10 minutos. A avaliação ocorreu na própria escola, durante o período escolar regular, no segundo semestre do ano letivo, e foi conduzida por três pesquisadoras (uma doutoranda; duas mestrandas). Aos pais e professores foram entregues o IFEI e MTA-SNAP-IV, sendo solicitado que respondessem e devolvessem os questionários aos pesquisadores até o término da coleta. Foram providas instruções claras, por escrito, para preenchimento e entrega das escalas. As respostas aos instrumentos ocorreram de forma autônoma, sem a presença ou o auxílio dos pesquisadores. Apesar disso, as pesquisadoras permaneceram na escola durante todo período de coleta e se disponibilizaram para esclarecer dúvidas de pais e professores e auxiliar no preenchimento das escalas quando preciso. Houve retorno de $93,05 \%$ das escalas enviadas aos pais e $100 \%$ de retorno das escalas enviadas às professoras.

\section{Resultados}

Uma vez que os desempenhos dos participantes não se distribuíram em conformidade com a curva normal, foram conduzidas análises não-paramétricas. Utilizou-se a análise de correlação de Spearman para verificar a relação entre os escores no IFEI e no MTA-SNAP-IV respondidos por pais e por professores e análise de Wilcoxon para comparar respostas de pais e professores aos mesmos instrumentos. Após, procedeu-se à correlação de Spearman para verificar a relação entre respostas às escalas e desempenho no TSS. Foram conduzidas análises para a amostra como um todo e para cada nível escolar separadamente, de modo a permitir uma compreensão mais detalhada das relações entre as medidas nas diferentes séries avaliadas.

A Tabela 1 apresenta as relações entre os escores nas escalas (IFEI e MTA-SNAP-IV) respondidas por pais e professores. Houve maior número de correlações entre as respostas de pais e de professores nas medidas do MTA-SNAP-IV do que nas medidas do IFEI. Porém, mesmo considerando apenas o MTA-SNAP-IV, observou-se que as relações encontradas foram de baixas a moderadas (apenas uma correlação considerada alta foi identificada, no indicador 'hiperatividade' na $1^{\mathrm{a}}$ fase, com $r=0,67 ; p \leq$ $0,001)$. No IFEI, apenas a pontuação na escala 'Controle Inibitório' apresentou relação entre relatos de pais e professores na $1^{\text {a }}$ fase e amostra total. Os resultados apontaram também para uma tendência de decréscimo no número de relações com a progressão escolar.

A Tabela 2 apresenta as estatísticas descritivas e o $Z$ de Wilcoxon. Algumas diferenças significativas entre pontuações atribuídas por pais e professores foram identificadas em ambas as escalas, IFEI e MTA-SNAP-IV. Em todos os casos, tanto considerando a amostra geral ou os níveis escolares específicos, os pais atribuíram maiores pontuações, isto é, avaliaram as crianças como possuindo maiores dificuldades em relação à avaliação dos professores. 
Tabela 1

Matriz de Correlações de Spearman entre os Escores no IFEI e no MTA-SNAP-IV Respondidos por Pais e por Professores

\begin{tabular}{|c|c|c|c|c|c|c|c|}
\hline & \multicolumn{7}{|c|}{ Correlações entre pontuações de pais e de professores } \\
\hline & \multicolumn{5}{|c|}{ IFEI } & \multicolumn{2}{|c|}{ MTA-SNAP-IV } \\
\hline & $\mathrm{CI}$ & MT & PL & $\mathrm{RG}$ & Tot & Des & Hip \\
\hline Geral & $0,25^{*}$ & 0,09 & 0,10 & 0,18 & 0,19 & $0,36^{* *}$ & $0,39^{* *}$ \\
\hline $1^{\mathrm{a}}$ Fase & $0,43^{*}$ & 0,15 & 0,29 & 0,24 & 0,22 & $0,44^{*}$ & $0,67 * *$ \\
\hline $2^{\mathrm{a}}$ Fase & 0,26 & 0,20 & 0,13 & 0,04 & 0,28 & $0,39^{*}$ & 0,27 \\
\hline $1^{\circ}$ Ano & 0,08 & 0,03 & $-0,04$ & 0,22 & 0,10 & 0,29 & 0,12 \\
\hline
\end{tabular}

Nota. CI: controle inibitório; MT: memória de trabalho; PL: planejamento; RG: regulação; Tot: total; Des: Desatenção; Hip: Hiperatividade.

$* p \leq 0,05 ; * * p \leq 0,01$.

Tabela 2

Estatísticas Descritivas e Z de Wilcoxon no IFEI e MTA-SNAP-IV Respondidos por Pais e Professores para a Amostra Total e por Nível Escolar

\begin{tabular}{|c|c|c|c|c|c|c|c|c|c|c|c|c|c|c|}
\hline & \multicolumn{10}{|c|}{$\begin{array}{c}\text { IFEI } \\
M(D P)\end{array}$} & \multicolumn{4}{|c|}{$\begin{array}{c}\text { MTA-SNAP-IV } \\
M(D P)\end{array}$} \\
\hline & \multicolumn{2}{|c|}{ CI } & \multicolumn{2}{|c|}{ MT } & \multicolumn{2}{|c|}{ PL } & \multicolumn{2}{|c|}{ RG } & \multicolumn{2}{|c|}{ Tot } & \multicolumn{2}{|c|}{ Des } & \multicolumn{2}{|c|}{ Hip } \\
\hline & Pais & Profs & Pais & Profs & Pais & Profs & Pais & Profs & Pais & Profs & Pais & Profs & Pais & Profs \\
\hline Geral & $\begin{array}{l}16,55 \\
(4,82)\end{array}$ & $\begin{array}{c}13,9 \\
(5,25)\end{array}$ & $\begin{array}{l}20,12 \\
(6,82)\end{array}$ & $\begin{array}{l}19,87 \\
(8,16)\end{array}$ & $\begin{array}{c}8,92 \\
(3,36)\end{array}$ & $\begin{array}{c}8,89 \\
(3,56)\end{array}$ & $\begin{array}{l}13,95 \\
(4,16)\end{array}$ & $\begin{array}{l}11,76 \\
(4,56)\end{array}$ & $\begin{array}{c}62,04 \\
(17,25)\end{array}$ & $\begin{array}{c}56,89 \\
(21,43)\end{array}$ & $\begin{array}{c}8,11 \\
(6,97)\end{array}$ & $\begin{array}{c}5,67 \\
(6,15)\end{array}$ & $\begin{array}{c}8,32 \\
(5,99)\end{array}$ & $\begin{array}{c}4,56 \\
(6,13)\end{array}$ \\
\hline$Z$ & \multicolumn{2}{|c|}{$-3,573$} & \multicolumn{2}{|c|}{$-0,549$} & \multicolumn{2}{|c|}{$-0,373$} & \multicolumn{2}{|c|}{$-3,629$} & \multicolumn{2}{|c|}{$-1,514$} & \multicolumn{2}{|c|}{$-2,985$} & \multicolumn{2}{|c|}{$-5,705$} \\
\hline$p$ & \multicolumn{2}{|c|}{$<0,001$} & \multicolumn{2}{|c|}{0,583} & \multicolumn{2}{|c|}{0,709} & \multicolumn{2}{|c|}{$<0,001$} & \multicolumn{2}{|c|}{0,130} & \multicolumn{2}{|c|}{0,003} & \multicolumn{2}{|c|}{$<0,001$} \\
\hline $\begin{array}{c}1^{\mathrm{a}} \\
\text { Fase }\end{array}$ & $\begin{array}{l}17,31 \\
(5,51)\end{array}$ & $\begin{array}{l}15,32 \\
(5,79)\end{array}$ & $\begin{array}{l}20,14 \\
(8,06)\end{array}$ & $\begin{array}{l}23,21 \\
(9,65)\end{array}$ & $\begin{array}{c}8,86 \\
(3,56)\end{array}$ & $\begin{array}{l}10,24 \\
(3,92)\end{array}$ & $\begin{array}{l}14,11 \\
(4,61)\end{array}$ & $\begin{array}{l}13,08 \\
(4,89)\end{array}$ & $\begin{array}{c}64,69 \\
(20,03)\end{array}$ & $\begin{array}{c}66,79 \\
(24,83)\end{array}$ & $\begin{array}{c}5,5 \\
(4,75)\end{array}$ & $\begin{array}{c}7,68 \\
(7,48)\end{array}$ & $\begin{array}{c}9,44 \\
(6,57)\end{array}$ & $\begin{array}{c}7,13 \\
(7,69)\end{array}$ \\
\hline$Z$ & \multicolumn{2}{|c|}{$-2,646$} & \multicolumn{2}{|c|}{$-0,883$} & \multicolumn{2}{|c|}{$-1,094$} & \multicolumn{2}{|c|}{$-1,842$} & \multicolumn{2}{|c|}{$-0,265$} & \multicolumn{2}{|c|}{$-0,565$} & \multicolumn{2}{|c|}{$-2,406$} \\
\hline$p$ & \multicolumn{2}{|c|}{0,008} & \multicolumn{2}{|c|}{0,377} & \multicolumn{2}{|c|}{0,274} & \multicolumn{2}{|c|}{0,065} & 0,7 & 991 & 0,5 & 572 & 0,0 & 16 \\
\hline $\begin{array}{c}2^{\mathrm{a}} \\
\text { Fase }\end{array}$ & $\begin{array}{l}15,47 \\
(3,75)\end{array}$ & $\begin{array}{l}13,94 \\
(4,97)\end{array}$ & $\begin{array}{l}19,64 \\
(6,34)\end{array}$ & $\begin{array}{l}18,70 \\
(6,9)\end{array}$ & $\begin{array}{c}9,0 \\
(3,48)\end{array}$ & $\begin{array}{c}8,3 \\
(3,09)\end{array}$ & $\begin{array}{l}13,83 \\
(3,54)\end{array}$ & $\begin{array}{l}11,55 \\
(4,39)\end{array}$ & $\begin{array}{c}62,28 \\
(15,55)\end{array}$ & $\begin{array}{c}56,45 \\
(18,72)\end{array}$ & $\begin{array}{c}5,56 \\
(4,36)\end{array}$ & $\begin{array}{c}4,97 \\
(6,35)\end{array}$ & $\begin{array}{c}7,28 \\
(4,94)\end{array}$ & $\begin{array}{c}4,12 \\
(6,44)\end{array}$ \\
\hline$Z$ & $-1,7$ & 794 & -0 , & 178 & -1 , & 970 & -2 & 60 & -1, & 442 & $-1, S$ & 911 & -3 & 319 \\
\hline$p$ & 0,0 & 73 & 0,8 & 59 & 0,2 & 85 & 0,0 & & 0,1 & 49 & 0,0 & 956 & 0,0 & 01 \\
\hline $\begin{array}{c}1^{\circ} \\
\text { Ano }\end{array}$ & $\begin{array}{c}16,73 \\
(4,9)\end{array}$ & $\begin{array}{c}13,16 \\
(5,0)\end{array}$ & $\begin{array}{c}20,38 \\
(6,4)\end{array}$ & $\begin{array}{l}18,68 \\
(7,45)\end{array}$ & $\begin{array}{c}8,9 \\
(3,22)\end{array}$ & $\begin{array}{c}8,46 \\
(3,43)\end{array}$ & $\begin{array}{l}13,92 \\
(4,28)\end{array}$ & $\begin{array}{l}11,18 \\
(4,38)\end{array}$ & $\begin{array}{c}60,35 \\
(16,53)\end{array}$ & $\begin{array}{c}51,93 \\
(19,04)\end{array}$ & $\begin{array}{l}11,76 \\
(8,17)\end{array}$ & $\begin{array}{l}4,95 \\
(5,1)\end{array}$ & $\begin{array}{c}8,27 \\
(6,18)\end{array}$ & $\begin{array}{l}3,43 \\
(4,6)\end{array}$ \\
\hline$Z$ & $-1,8$ & 851 & -0 & 234 & -0 & 271 & -2 & 10 & -1, & 076 & $-3,6$ & 658 & -3, & 947 \\
\hline$p$ & 0,0 & 64 & 0,8 & 15 & 0,7 & 86 & 0,0 & & 0,2 & 82 & $<0$ &, 001 & $<0$ & 001 \\
\hline
\end{tabular}

Nota. CI: controle inibitório; MT: memória de trabalho; PL: planejamento; RG: regulação; Tot: total; Des: Desatenção; Hip: Hiperatividade. 
Tabela 3

Matriz de Correlações de Spearman entre os Desempenhos no Teste de Stroop e os Desempenhos no IFEI e no MTA-SNAP-IV, Respondidos por Pais e Professores para $1^{\text {a }}$ Fase

Teste de Stroop Semântico

\begin{tabular}{|c|c|c|c|c|c|c|c|c|}
\hline & & & \\
\hline & & & \multicolumn{2}{|c|}{ Parte 1} & \multicolumn{2}{|c|}{ Parte 2} & \multicolumn{2}{|c|}{$\begin{array}{c}\text { Interferência } \\
\text { (parte } 2-\text { parte } 1)\end{array}$} \\
\hline & & & Escore & $\mathrm{TR}$ & Escore & $\mathrm{TR}$ & Escore & TR \\
\hline \multirow[t]{7}{*}{ Pais } & \multirow[t]{5}{*}{ IFEI } & $\mathrm{CI}$ & $-0,34$ & $-0,38^{*}$ & $-0,003$ & $-0,002$ & 0,094 & 0,27 \\
\hline & & MT & $-0,31$ & $-0,14$ & $-0,02$ & 0,02 & $-0,001$ & 0,06 \\
\hline & & PL & $-0,29$ & $-0,13$ & 0,05 & $-0,08$ & 0,09 & $-0,02$ \\
\hline & & $\mathrm{RG}$ & $-0,26$ & $-0,29$ & 0,10 & $-0,18$ & 0,12 & 0,02 \\
\hline & & Tot & $-0,36^{*}$ & $-0,29$ & 0,004 & $-0,09$ & 0,04 & 0,09 \\
\hline & \multirow[t]{2}{*}{ SNAP } & Des & $-0,31$ & $-0,07$ & 0,06 & $-0,15$ & 0,08 & $-0,04$ \\
\hline & & Hip & $-0,30$ & $-0,32$ & $-0,07$ & $-0,07$ & 0,08 & 0,21 \\
\hline \multirow[t]{7}{*}{ Professores } & \multirow[t]{5}{*}{ IFEI } & $\mathrm{CI}$ & $-0,27$ & 0,07 & $-0,17$ & $-0,22$ & 0,06 & $-0,16$ \\
\hline & & MT & $-0,38^{*}$ & 0,18 & $-0,25$ & $-0,16$ & $-0,01$ & $-0,16$ \\
\hline & & PL & $-0,40^{*}$ & 0,01 & $-0,27$ & $-0,26$ & 0,06 & $-0,17$ \\
\hline & & $\mathrm{RG}$ & $-0,22$ & $-0,05$ & $-0,21$ & $-0,19$ & $-0,04$ & $-0,07$ \\
\hline & & Tot & $-0,34$ & 0,13 & $-0,25$ & $-0,25$ & 0,01 & $-0,22$ \\
\hline & \multirow[t]{2}{*}{ SNAP } & Des & $-0,25$ & $-0,09$ & $-0,19$ & 0,07 & $-0,03$ & 0,11 \\
\hline & & Hip & $-0,18$ & $-0,15$ & 0,04 & $-0,03$ & 0,24 & 0,14 \\
\hline
\end{tabular}

Nota. TR: tempo de reação. CI: controle inibitório; MT: memória de trabalho; PL: planejamento; RG: regulação; Tot: total; Des: Desatenção; Hip: Hiperatividade.

$* p \leq 0,05$.

Foram também conduzidas análises de correlação de Spearman entre o desempenho das crianças no TSS e as pontuações nas escalas preenchidas por ambos, pais ou professores. As Tabelas 3, 4 e 5 apresentam estes resultados em cada nível escolar.

Como apresentado na Tabela 3, conforme resposta dos pais, houve relações significativas entre a pontuação em controle inibitório do IFEI e o tempo de reação na parte congruente do TSS, assim como entre a pontuação total no IFEI e escore da parte 1 do TSS. Considerando os professores, houve relação significativa entre os indicadores de memória de trabalho e planejamento do IFEI com o escore da parte 1 do TSS.

A Tabela 4 ilustra os resultados das crianças da $2^{\mathrm{a}}$ fase. De acordo com respostas dos pais, houve relações significativas entre as subescalas memória de trabalho, controle inibitório, plane- jamento e total do IFEI com o tempo de reação na parte 2 do TSS. As subescalas planejamento e total no IFEI relacionaram-se ao tempo de reação na parte 1 do TSS. Considerando a perspectiva dos professores, foram observadas correlações significativas entre escores em planejamento e memória de trabalho no IFEI e desempenho na parte 1 do TSS. Aqui, observou-se também relação entre o indicador de desatenção do MTA-SNAP e os escores na parte 1 e de interferência do TSS.

Conforme apresentado na Tabela 5 , no $1^{\circ}$ ano, nenhuma relação foi evidenciada com os indicadores do MTA-SNAP-IV. Considerando o IFEI preenchido por pais, houve correlação entre a subescala de controle inibitório e o tempo de reação de interferência no TSS, e entre regulação e tempo de reação na parte 2 do TSS. Para os professores, o controle inibitório foi a única 
Tabela 4

Matriz de Correlações de Spearman entre os Desempenhos no Teste de Stroop e os Desempenhos no IFEI e no MTA-SNAP-IV, Respondidos por Pais e Professores para $2^{\text {a }}$ Fase

\begin{tabular}{|c|c|c|c|c|c|c|c|c|}
\hline & & & \multicolumn{6}{|c|}{ Teste de Stroop Semântico } \\
\hline & & & \multicolumn{2}{|c|}{ Parte 1} & \multicolumn{2}{|c|}{ Parte 2} & \multicolumn{2}{|c|}{$\begin{array}{c}\text { Interferência } \\
\text { (parte } 2-\text { parte } 1)\end{array}$} \\
\hline & & & Escore & TR & Escore & TR & Escore & TR \\
\hline \multirow[t]{7}{*}{ Pais } & IFEI & $\mathrm{CI}$ & 0,03 & $-0,27$ & $-0,11$ & $-0,50 * *$ & $-0,14$ & $-0,29$ \\
\hline & & MT & $-0,11$ & $-0,31$ & $-0,03$ & $-0,36^{*}$ & $-0,05$ & $-0,06$ \\
\hline & & PL & $-0,18$ & $-0,51 * *$ & 0,04 & $-0,52 * *$ & 0,09 & $-0,10$ \\
\hline & & RG & 0,16 & $-0,27$ & 0,12 & $-0,21$ & $-0,02$ & $-0,01$ \\
\hline & & Tot & $-0,08$ & $-0,39^{*}$ & $-0,01$ & $-0,45^{*}$ & $-0,04$ & $-0,10$ \\
\hline & SNAP & Des & $-0,18$ & $-0,28$ & $-0,17$ & $-0,26$ & $-0,09$ & 0,02 \\
\hline & & Hip & 0,19 & $-0,12$ & 0,02 & $-0,01$ & $-0,16$ & 0,14 \\
\hline \multirow[t]{7}{*}{ Professores } & IFEI & $\mathrm{CI}$ & $-0,10$ & $-0,23$ & $-0,07$ & $-0,14$ & 0,12 & $-0,05$ \\
\hline & & MT & $-0,40^{*}$ & 0,03 & $-0,36$ & 0,09 & $-0,01$ & 0,11 \\
\hline & & PL & $-0,43^{*}$ & $-0,02$ & $-0,29$ & 0,05 & 0,07 & 0,11 \\
\hline & & $\mathrm{RG}$ & $-0,29$ & $-0,03$ & $-0,37$ & $-0,09$ & $-0,03$ & $-0,02$ \\
\hline & & Tot & $-0,34$ & $-0,10$ & $-0,31$ & $-0,08$ & 0,04 & 0,03 \\
\hline & SNAP & Des & $-0,50 * *$ & $-0,16$ & $-0,12$ & $-0,30$ & $0,39 *$ & $-0,09$ \\
\hline & & Hip & $-0,06$ & $-0,43^{*}$ & $-0,04$ & $-0,39 *$ & 0,11 & $-0,11$ \\
\hline
\end{tabular}

Nota. TR: tempo de reação. CI: controle inibitório; MT: memória de trabalho; PL: planejamento; RG: regulação; Tot: total; Des: Desatenção; Hip: Hiperatividade.

$* p \leq 0,05 ; * * p \leq 0,01$.

medida do IFEI que apresentou correlação com escore da parte 2 do teste de desempenho. Vale destacar que a avaliação feita por pais na subescala de controle inibitório do IFEI foi o único índice que se relacionou com alguma medida do TSS em todos os níveis escolares.

\section{Discussão}

O primeiro objetivo do estudo foi verificar a concordância entre pais e professores com relação à avaliação das funções executivas e dos sinais de desatenção e hiperatividade. Foram evidenciadas relativamente poucas relações entre as medidas avaliadas por pais e por professores, e as relações encontradas tenderam a apresentar magnitudes de baixa a moderada. Este baixo grau de acordo entre pais e professores é consistente com a literatura (Coutinho et al., 2009; Homer et al., 2000; Major \& Seabra-Santos, 2013; Mitsis et al., 2000; Seabra-Santos \& Gaspar, 2012; Serra-Pinheiro et al., 2008; Zucker et al., 2002). Interessante observar que estes autores avaliaram diferentes construtos, porém seus resultados convergem em relação a um mesmo padrão global de concordância baixa ou no máximo moderada entre informantes pais e professores.

Autores têm tentado explicar esta tendência a baixa concordância entre informantes acerca do comportamento infantil e juvenil como função das expectativas que cada avaliador tem em relação ao sujeito, dos julgamentos e interpreta- 
Tabela 5

Matriz de Correlações de Spearman entre os Desempenhos no Teste de Stroop e os Desempenhos no IFEI e no MTA-SNAP-IV, Respondidos por Pais e Professores para $1^{\circ}$ Ano

\begin{tabular}{|c|c|c|c|c|c|c|c|c|}
\hline & & & \multicolumn{6}{|c|}{ Teste de Stroop Semântico } \\
\hline & & & \multicolumn{2}{|c|}{ Parte 1} & \multicolumn{2}{|c|}{ Parte 2} & \multicolumn{2}{|c|}{$\begin{array}{c}\text { Interferência } \\
\text { (parte } 2-\text { parte } 1)\end{array}$} \\
\hline & & & Escore & TR & Escore & TR & Escore & TR \\
\hline \multirow[t]{7}{*}{ Pais } & IFEI & $\mathrm{CI}$ & 0,10 & 0,11 & $-0,24$ & $-0,34$ & $-0,28$ & $-0,42^{*}$ \\
\hline & & MT & 0,13 & $-0,05$ & $-0,11$ & 0,05 & $-0,31$ & 0,003 \\
\hline & & PL & 0,15 & 0,001 & $-0,01$ & $-0,27$ & $-0,22$ & $-0,25$ \\
\hline & & RG & 0,11 & $-0,13$ & $-0,10$ & $-0,37^{*}$ & $-0,22$ & $-0,23$ \\
\hline & & Tot & 0,12 & $-0,02$ & $-0,12$ & $-0,27$ & $-0,29$ & $-0,31$ \\
\hline & SNAP & Des & $-0,22$ & 0,33 & $-0,003$ & 0,27 & 0,15 & $-0,03$ \\
\hline & & Hip & $-0,18$ & 0,26 & $-0,12$ & 0,06 & $-0,01$ & $-0,21$ \\
\hline \multirow[t]{7}{*}{ Professores } & IFEI & $\mathrm{CI}$ & 0,06 & $-0,11$ & $0,28 *$ & $-0,15$ & 0,19 & $-0,04$ \\
\hline & & MT & 0,04 & $-0,05$ & 0,24 & $-0,16$ & 0,12 & $-0,14$ \\
\hline & & PL & 0,01 & $-0,06$ & 0,19 & $-0,11$ & 0,09 & $-0,05$ \\
\hline & & $\mathrm{RG}$ & 0,04 & $-0,04$ & 0,16 & $-0,19$ & 0,07 & $-0,13$ \\
\hline & & Tot & 0,03 & $-0,04$ & 0,24 & $-0,16$ & 0,13 & $-0,11$ \\
\hline & SNAP & Des & $-0,09$ & 0,01 & 0,20 & $-0,13$ & 0,19 & $-0,12$ \\
\hline & & Hip & $-0,01$ & 0,05 & 0,12 & $-0,03$ & 0,05 & $-0,04$ \\
\hline
\end{tabular}

Nota. TR: tempo de reação. CI: controle inibitório; MT: memória de trabalho; PL: planejamento; RG: regulação; Tot: total; Des: Desatenção; Hip: Hiperatividade.

$* p \leq 0,05$.

ções divergentes dos itens das escalas utilizadas e do próprio ambiente em que a observação ocorre (Homer et al., 2000; Major \& Seabra-Santos, 2013; Zucker et al., 2002). Conforme sugerido por Seabra-Santos e Gaspar (2012), as diferenças entre o ambiente em casa e o da escola poderiam induzir a ocorrência de comportamentos diferentes que, então, poderiam ser observáveis por um dos informantes, ainda que não pelo outro. É fato que pais e professores lidam com a criança em contextos bastante diferenciados em termos de estímulos, estrutura, regras, entre outros. Neste sentido, o baixo grau de concordância não deve ser visto necessariamente como negativo, mas como complementar. Ou seja, distintos ambientes e informantes podem permitir diferentes possibilidades de informação sobre a criança (Major \& Seabra-Santos, 2013).
Outro ponto de interesse refere-se à variação com relação ao grau de concordância em diferentes subescalas. Por exemplo, pais e professores tiveram maior acordo com relação à avaliação do controle inibitório das crianças pela IFEI. Porém nenhuma relação foi encontrada nas demais subescalas do IFEI, memória de trabalho, regulação, planejamento, ou mesmo no escore total. Já com relação à MTA-SNAP-IV, apesar de variabilidade dos coeficientes, houve correlações baixas em todas as subescalas para a amostra geral e as correlações foram de moderadas a altas se consideradas as crianças da $1^{\text {a }}$ fase. Essa variação no grau de concordância em diferentes medidas pode ser devida à natureza do construto avaliado e/ou à dificuldade de observá-lo e mensurá-lo. Por exemplo, considerando a faixa etária das crianças participantes deste estudo (de 
4 a 7 anos), pode ser mais fácil a pais e professores observar e pontuar itens relacionados ao controle inibitório, como 'Age de um modo mais desenfreado ou sem limites quando comparado(a) com outras crianças em um grupo', do que itens que envolvem comportamentos mais complexos ou mais difíceis de avaliar como aqueles das outras escalas, a exemplo da escala planejamento, 'Tem dificuldade em planejar uma ativida$d e$ '. Essa variação no grau de concordância em função do construto também ficou evidente nos dados de Seabra-Santos e Gaspar (2012). Os autores encontraram relações variadas entre pais e professores nas dimensões relacionamento, comunicação e aprendizagem, porém relação nula na dimensão autorregulação. Destaca-se, ainda, que a compreensão dos itens das escalas depende das características dos respondentes (Major \& Seabra-Santos, 2013). Portanto, é possível que os pais da presente amostra, proveniente de escola pública, tenham tido uma interpretação diferente da feita pelos professores, o que pode ajudar a entender as diferenças nos relatos feitos.

Além da variabilidade do grau de concordância nas diferentes dimensões avaliadas, o estudo também mostrou que o acordo de pais e professores diferiu como função do nível escolar. Maior número de relações entre pontuações de pais e professores foi identificado quando consideradas as crianças mais jovens, da $1^{\text {a }}$ fase (controle inibitório do IFEI; desatenção e hiperatividade da MTA-SNAP-IV), com diminuição das relações encontradas na $2^{\text {a }}$ fase (desatenção da MTA-SNAP-IV) e nenhuma relação significativa observada no $1^{\circ}$ ano. Uma possível hipótese para este ocorrido seria que as demandas da escola e de casa vão se tornando progressivamente mais distintas, isto é, o que se passa a esperar da criança no ambiente escolar torna-se bastante diferente do que se espera dela em casa, sobretudo a partir do ingresso no ambiente mais estruturado do Ensino Fundamental.

Complementado estas informações, evidenciou-se que os pais atribuíram a seus filhos mais sinais de desatenção e hiperatividade, assim como maiores dificuldades em controle inibitório e regulação. Apesar da amostra deste estudo ser não-clínica, os resultados foram semelhantes aos obtidos por Coutinho et al. (2009) em crianças e adolescentes com TDAH, o que levou os autores a concluir que pais apresentaram maior queixa sobre o comportamento dos filhos. De fato, uma possível fonte de divergência entre informes, neste caso entre pais e professores, refere-se à tendência de um informante ser muito benevolente ou crítico. Outras fontes de divergência referem-se aos distintos contextos (casa e escola) que embasaram a avaliação de pais e professores e ao conhecimento que cada informante detém sobre o comportamento (o que seria ou não adequado para determinada faixa etária) em avaliação (Major \& Seabra-Santos, 2013). Neste sentido, uma possível hipótese para compreender este resultado seria a de que os pais possuem uma tendência a superestimar dificuldades de comportamento das crianças e o professor, pelo maior conhecimento sobre desenvolvimento infantil e presença de referencial para comparação (outras crianças), estaria em melhores condições para a avaliação da criança.

O segundo objetivo do estudo foi investigar as relações entre as pontuações nas escalas (IFEI e MTA-SNAP-IV) e o desempenho das crianças em um teste de inibição, o Teste de Stroop Semântico. Estudo prévio também conduzido com pré-escolares evidenciou algumas correlações, apesar de baixas, entre desempenho em testes de funções executivas e indicadores de desatenção e hiperatividade (Pereira et al., 2012). Porém, ao contrário destes achados, no presente estudo poucas relações foram identificadas e, dentre as identificadas, houve algumas inconsistências. Por exemplo, apenas na $2^{\mathrm{a}}$ fase foram identificadas relações entre desempenho em medidas do Teste de Stroop Semântico e pontuações na MTA-SNAP-IV pelos professores. As correlações encontradas apontaram que crianças mais desatentas apresentaram tendência a cometer mais erros na parte 1 do teste. Isso poderia ser explicado, entre outras hipóteses, pela novidade inicial da tarefa, talvez estas crianças demorem um pouco mais para conseguir se engajar na atividade, por exemplo. Porém, este pior desempenho na parte 1 , quando aplicado à fórmula 'desempenho na parte 2 - desempenho na parte 1' para extração do escore de interferência, levou 
a um efeito superestimado (mais positivo, indicando menor conflito). Este resultado também se relacionou à escala de desatenção pelos professores e, dessa forma, as crianças avaliadas como mais desatentas tenderam a apresentar menor efeito de interferência. Apenas as relações entre sinais de hiperatividade e tempo de reação nas partes 1 e 2 do TSS apresentam consistência teórica, sugerindo que as crianças com mais sinais de hiperatividade tenderam a ser mais rápidas em ambas as partes do instrumento, possivelmente respondendo de forma mais impulsiva, consistente com alguns achados de Pereira et al. (2012).

Outro aspecto refere-se à relação entre as medidas do teste de desempenho e do IFEI. Poucas correlações foram encontradas entre as medidas e todas com magnitude de baixa a moderada. De fato, outra investigação também encontrou apenas correlação moderada entre uma dimensão cognitiva avaliada por resposta de pais e professores e o desempenho em teste de QI (Seabra-Santos \& Gaspar, 2012), sugerindo que as escalas, longe de substituírem os testes de desempenho, seriam uma ferramenta que permitia uma aproximação grosseira do desempenho da criança.

Uma breve interpretação dos resultados encontrados mostra que, na $1^{\text {a }}$ fase, crianças avaliadas pelos pais com maiores dificuldades em controle inibitório tenderam a ser mais rápidas na parte 1 do TSS, talvez por responderem mais impulsivamente. Também aquelas avaliadas com maiores dificuldades em funcionamento executivo total cometeram mais erros também na parte 1 do teste. $\mathrm{Na}$ avaliação dos professores, crianças com escores piores em memória de trabalho ou planejamento tenderam a cometer também mais erros na mesma parte do TSS, o que também foi observado na $2^{\mathrm{a}}$ fase. Neste nível, crianças avaliadas pelos pais com maiores dificuldades em memória de trabalho ou controle inibitório tenderam a responder mais rapidamente à segunda parte do TSS, enquanto que aquelas avaliadas com mais dificuldades em planejamento e funcionamento executivo total apresentaram esse padrão de resposta mais rápido em ambas as partes, 1 e 2, do teste. Uma hipótese explicativa para tais resultados é a de que essas crianças tendem a ponderar menos antes de emitir uma resposta, o que explicaria seu menor tempo de reação. Já no $1^{\circ}$ ano, crianças avaliadas pelos pais com maiores dificuldades em controle inibitório tenderam a apresentar maior tempo para a solução do conflito imposto pelo TSS, sofrendo maior efeito de interferência; aquelas com maior dificuldade em regulação tenderam a demandar mais tempo na parte 2 , incongruente, do teste. Por outro lado, na avaliação dos professores, as crianças consideradas com maiores dificuldade de controle inibitório tenderam a ter maiores escores na parte 2 do TSS, este último sendo um resultado inesperado e teoricamente inconsistente. É possível que este resultado reflita uma avaliação menos apurada da habilidade de controle inibitório pelos professores, no entanto esta hipótese carece de maior respaldo, uma vez que esse achado ocorreu somente neste nível escolar.

Um aspecto interessante a ser notado, porém, é que, no curso dos níveis escolares sucessivos, as correlações das subescalas do IFEI passaram a ocorrer com medidas mais complexas do teste de desempenho. Assim, na $1^{\text {a }}$ fase, as habilidades executivas avaliadas por pais e professores relacionaram-se com escore e tempo de reação na parte 1 do TSS, que apenas exige a nomeação de figuras. $\mathrm{Na} 2^{\mathrm{a}}$ fase, apesar de a correlação ainda se estabelecer com escore e tempo da parte 1, outras, sobretudo considerando avaliação dos pais, se estabeleceram com o tempo de reação da parte 2 . Por fim, no $1^{\circ}$ ano, as relações encontradas foram entre medidas do IFEI e escore e tempo na parte 2 e interferência do teste de desempenho, sugerindo que crianças com melhores habilidades de regulação, conforme resposta de pais, e inibição, conforme pais e professores, desempenham-se melhor no Teste de Stroop. Este ocorrido pode ser devido a uma avaliação mais adequada por parte de pais e professores com o crescimento das crianças, por exemplo, em função da maior possibilidade de observação em tarefas mais diversificadas, ou atrelada ao próprio desenvolvimento das funções executivas das crianças. De fato, apesar do longo curso de desenvolvimento destas habilidades, há evidências de maior efeito de idade entre aproximadamente os cinco e sete anos (Best, Miller, \& Naglieri, 2011), sendo que, entre os quatro 
e cinco anos, as crianças tornam-se progressivamente mais hábeis em focalizar a atenção e controlar seu comportamento (Dawson \& Guare, 2010; García-Molina et al., 2009). Assim, o rápido desenvolvimento destas habilidades nesta faixa etária pode ter se refletido no desempenho das crianças em partes mais complexas do TSS, assim como na avaliação por pais e professores.

Uma última questão é referente a se escala $\mathrm{e}$ teste estariam de fato avaliando o mesmo construto. Ou seja, medidas de desempenho, a exemplo do Teste de Stroop Semântico, oferecem uma condição padronizada e estruturada. Ao contrário, escalas são medidas funcionais, destinadas a mensurar o funcionamento da criança no dia-a-dia em ambientes muito menos estruturados do que uma situação de testagem. Investigações acerca desse argumento precisam ser realizadas. Neste ponto, cabe destacar que, apesar da variação das correlações estabelecidas nos diferentes níveis escolares, a pontuação atribuída pelos pais na subescala de controle inibitório foi a única que se relacionou com alguma medida do TSS em todos os níveis escolares. Esse resultado é coerente, sobretudo se considerados $2^{\mathrm{a}}$ fase e $1^{\mathrm{o}}$ ano (devido às relações com índices da parte 2 e de interferência do TSS), uma vez que o teste de desempenho utilizado demanda habilidade de inibição para sua resolução (Berwid et al., 2005). Assim, as duas medidas de inibição, escala e teste de desempenho, mostraram-se relacionadas entre si com magnitude de baixa a moderada.

Há uma tendência em se esperar resultados relativamente uniformes ao utilizar diferentes medidas, a exemplo de testes de desempenho e escalas, que avaliem o mesmo construto ou construtos relacionados. Os resultados aqui encontrados, com correlações baixas e moderadas entre as duas formas de avaliação, sugerem que tais medidas podem ser compreendidas como complementares ao derivar diferentes informações, com base na habilidade e no funcionamento em diferentes contextos.

\section{Considerações Finais}

De forma geral, o estudo mostrou que há alguma concordância, em geral baixa, entre infor- mantes, pais e professores, com relação à avaliação do funcionamento executivo e de indicadores de desatenção e hiperatividade de crianças pré-escolares e ingressantes no Ensino Fundamental, sendo que pais avaliaram as crianças como possuindo mais dificuldades. Houve maior grau de concordância entre os respondentes em medidas específicas, como em controle inibitório e nos indicadores de desatenção e hiperatividade, porém apenas nas crianças mais jovens. Poucas correlações consistentes foram observadas entre as pontuações nas escalas e medidas em um teste de desempenho. Entre estas, destaca-se a relação entre avaliação do controle inibitório por pais e desempenho das crianças no TSS. As relações, no entanto, não foram altas, apontando uma convergência entre as medidas, mas também sugerindo que ambas mensuram aspectos específicos.

Limitações do estudo incluem a ausência de controle do gênero e do respondente específico das escalas enviadas para preenchimento pelos pais, ou seja, pai ou mãe, bem como acompanhamento da leitura e da compreensão dos itens pelos respondentes. Também se pode mencionar o uso de um único teste de desempenho, bem como a avaliação de um único componente específico das funções executivas. Sugere-se que estudos futuros investiguem o grau de concordância entre pai e mãe na avaliação de crianças, investiguem o efeito da escolaridade dos pais sobre a resposta às escalas e utilizem outros testes de desempenho, que possibilitem uma avaliação mais ampla das funções executivas ou outros construtos relevantes. Estes estudos permitirão aprofundar a compreensão atual acerca das relações entre desempenho em testes e em escalas funcionais, sugerindo instrumentos com maior validade ecológica e esclarecendo as diferenças entre estas avaliações. No que tange às implicações do presente estudo para a avaliação das funções executivas em pré-escolares e ingressantes no Ensino Fundamental, destaca-se a necessidade do recurso a diversas fontes de informação. Ou seja, a baixa convergência entre as fontes, pais e professores, escalas e testes de desempenho, reforça a necessidade de que todos sejam considerados em conjunto no processo de avaliação infantil. 


\section{Referências}

Baddeley, A. (2000). The episodic buffer: A new component of working memory? Trends in Cognitive Sciences, 4(11), 417-423. doi:10.1016/ S1364-6613(00)01538-2

Barkley, R. A. (1997). Behavioral inhibition, sustained attention and executive function: Constructing a unifying theory of ADHD. Psychological Bulletin, 121, 65-94. doi:10.1037/0033-2909.121.1.65

Berwid, O., Kera, E. C., Santra, A., Bender, H., \& Halperin, J. (2005). Sustained attention and response inhibition in preschool children at-risk for AD/HD. Journal of Child Psychology \& Psychiatry, 46(11), 1219-1229. doi:10.1111/ j.1469-7610.2005.00417.x

Best, J. R., Miller, P., \& Naglieri, J. A. (2011). Relations between executive function and academic achievement from ages 5 to 17 in a large, representative national sample. Learning and Individual Differences, 21(4), 327-336. doi:10.1016/j. lindif.2011.01.007

Blair, C., \& Diamond, A. (2008). Biological processes in prevention and intervention: The promotion of self-regulation as a means of preventing school failure. Development and Psychopathology, 20, 899-891. doi: 10.1017/S0954579408000436

Blair, C., \& Razza, R. P. (2007). Relating effortful control, executive function, and false-belief understanding to emerging math and literacy ability in kindergarten. Child Development, 78, 647663. doi:10.1111/j.1467-8624.2007.01019.x

Capovilla, A. G. S., \& Dias, N. M. (2008). Desenvolvimento de habilidades atencionais em estudantes da $1^{\mathrm{a}}$ à $4^{\mathrm{a}}$ sério do ensino fundamental e relação com rendimento escolar. Psicopedagogia, 25(78), 198-211.

Coutinho, G., Mattos, P., Schmitz, M., Fortes, D., \& Borges, M. (2009). Concordância entre relato de pais e professores para sintomas de TDAH: Resultados de uma amostra clínica brasileira. Revista de Psiquiatria Clínica, 36(3), 97-100. doi:10.1590/S0101-60832009000300003

Dawson, P., \& Guare, R. (2010). Executive skills in children and adolescents: A practical guide to assessment and intervention. New York: The Guilford Press.

Diamond, A. (2013). Executive functions. Annual Review of Psychology, 64, 135-168. doi:10.1146/ annurev-psych-113011-143750
Dias, N. M., \& Seabra, A. G. (2013). Funções executivas: Desenvolvimento e intervenção. Temas sobre Desenvolvimento, 19(107), 206-212.

Fonseca, R. P., Oliveira, C. R., Gindri, G., Zimmermann, N., Reppold, C. T., \& Parente, M. A. M. P. (2010). Teste Hayling: Um instrumento de avaliação de componentes das funções executivas. In C. Hutz (Ed.), Avanços em Avaliação Psicológica e Neuropsicológica de crianças e adolescentes (pp. 337-364). São Paulo, SP: Casa do Psicólogo.

García-Molina, A., Enseñat-Cantallops, A., Tirapu-Ustárroz, J., \& Roig-Rovira, T. (2009). Maduración de la corteza prefrontal y desarrollo de las funciones ejecutivas durante los primeros cinco años de vida. Revista Neurologia, 48, 435-440.

Gazzaniga, M. S., Ivry, R. B., \& Mangun, G. R. (2006). Neurociência cognitiva. Porto Alegre, RS: Artmed.

Gonçalves, H. A., Mohr, R. M., Moraes, A. L., Siqueira, L. S., Prando, M. L., \& Fonseca, R. P. (2013). Componentes atencionais e de funções executivas em meninos com TDAH: Dados de uma bateria neuropsicológica flexível. Jornal Brasileiro de Psiquiatria, 62, 13-21. doi:10.1590/S0047-20852013000100003

Guilherme, P. R., Mattos, P., Serra-Pinheiro, M. A., \& Regalla, M. A. (2007). Conflitos conjugais e familiares e presença de transtorno de déficit de atenção e hiperatividade (TDAH) na prole: Revisão sistemática. Jornal Brasileiro de Psiquiatria, 56(3), 201-207. doi:10.1590/S004720852007000300008

Homer, C. J., Baltz, R. D., Hickson, G. B., Miles, P. V., Newman, T. B., Shook, J. E., \& Zurhellen, W. M. (2000). Clinical practice guideline: Diagnosis and evaluation of the child with attentiondeficit/hyperactivity disorder. American Academy of Pediatrics, 105(5), 1158-1170.

Lezak, M. D., Howieson, D. B., \& Loring, D. W. (2004). Neuropsychological assessment $\left(4^{\text {th }} \mathrm{ed}\right.$.). New York: Oxford University Press.

Major, S., \& Seabra-Santos, M. J. (2013). Uso de inventários comportamentais para a avaliação socioemocional em idade pré-escolar. Avaliação Psicológica, 12(1), 101-107.

Malloy-Diniz, L. F., Sedo, M., Fuentes, D., \& Leite, W. B. (2008). Neuropsicologia das funções executivas. In D. Fuentes, L. F. Malloy-Diniz, C. H. P. Camargo, \& R. M. Cosenza (Eds.), Neurop- 
sicologia teoria e prática (pp. 387-206). Porto Alegre, RS: Artmed.

Mattos, P., Serra-Pinheiro, M. A., Rohde, L. M., \& Pinto, D. (2006). Apresentação de uma versão em português para uso no Brasil do instrumento MTA-SNAP-IV de avaliação de sintomas de transtorno do déficit de atenção/hiperatividade e sintomas de transtorno desafiador e de oposição. Revista de Psiquiatria do Rio Grande do Sul, 28(3), 290-297.

Menezes, A., Godoy, S., Teixeira, M. C. T. V., Carreiro, L. R. R., \& Seabra, A. G. (2012). Definições teóricas acerca das funções executivas e da atenção. In A. G. Seabra \& N. M. Dias (Eds.), Avaliação neuropsicológica cognitiva: Atenção e funções executivas: Vol. 1 (pp. 34-41). São Paulo, SP: Memnon.

Mitsis, E. M., McKay, K. E., Schulz, K. P., Newcorn, J. H., \& Halperin, J. M. (2000). Parentteacher concordance for DSM-IV Attention Deficit/Hyperactive Disorder in a clinic-referred sample. Journal of the American Academy of Child \& Adolescent Psychiatry, 39(3), 308-313. doi:10.1097/00004583-200003000-00012

Miyake, A., Friedman, N. P., Emerson, M. J., Witzki, A. H., \& Howerter, A. (2000). The unity and diversity of executive functions and their contributions to complex "Frontal Lobe" tasks: A latent variable analysis. Cognitive Psychology, 41, 49100. doi:10.1006/cogp.1999.0734

Nigg, J. (2001). Is ADHD a Disinhibitory Disorder? Psychological Bulletin, 127, 571-598. doi:10.1037//0033-2909.127.5.571

Pereira, A. P. P., León, C., Dias, N. M., \& Seabra, A. G. (2012). Avaliação de crianças pré-escolares: Relação entre testes de funções executivas e indicadores de desatenção e hiperatividade. Revista Psicopedagogia, 29(90), 279-289.

Seabra-Santos, M. J., \& Gaspar, M. F. (2012). Pais, educadores e testes: Estão de acordo na avaliação de aptidões de crianças pré-escolares? Psicologia: Reflexão e Crítica, 25, 203-211.

Serra-Pinheiro, M. A., Mattos, P., \& Regalla, M. A. (2008). Inattention, hyperactivity, and oppositional-defiant Symptoms in Brazilian adolescents: Gender prevalence and agreement between teachers and parents in a non-English speaking population. Journal of Attention Disorders, 12(2), 135-140. doi:10.1177/1087054708314620
Thorell, L., \& Nyberg, L. (2008). The Childhood Executive Functioning Inventory (CHEXI): A new rating instrument for parents and teachers. Developmental Neuropsychology, 33, 536-552. doi: $10.1080 / 87565640802101516$.

Trevisan, B. T. (2010) Atenção e controle inibitório em pré-escolares e correlação com indicadores de desatenção e hiperatividade (Dissertação de mestrado, Programa de Pós-Graduação em Distúrbios do Desenvolvimento, Universidade Presbiteriana Mackenzie, São Paulo, SP, Brasil).

Trevisan, B. T., Dias, N. M., Prust, A. P., Martoni, A. T., Santana, T., \& Seabra, A. G. (2011). Tradução e adaptação da Childhood Executive Functioning Inventory (CHEXI) para o contexto brasileiro. In Anais do XXI Congresso Brasileiro da ABENEPI e I Congresso Internacional da $A B E N E P I$. São Paulo, SP: Associação Brasileira de Neurologia, Psiquiatria Infantil.

Trevisan, B. T., Dias, N. M., Berberian, A. A., \& Seabra, A. G. (no prelo). Childhood Executive Functioning Inventory: Adaptation and psychometric features of the Brazilian version. Psico-USF, 22(1)

Vitaro, F., Brendgen, M., Larose, S., \& Tremblay, R. E. (2005). Kindergarten disruptive behaviors, protective factors, and educational achievement by early adulthood. Journal of Educational Psychology, 97(4), 617-629. doi:10.1037/00220663.97.4.617

Willcutt, E. G., Doyle, A. E., Nigg, J. T., Faraone, S. V., \& Pennington, B. F. (2005). Validity of the executive function theory of attentiondeficit/hyperactivity disorder: A meta-analytic review. Biological Psychiatry, 57, 1336-1346. doi:10.1016/j.biopsych.2005.02.006

Zucker, M., Morris, M., Ingram, S. M., Morris, R. D., \& Bakeman, R. (2002). Concordance of self- and informant ratings of adults' current and childhood attention-deficit/hyperactivity disorder symptoms. Psychological Assessment, 14, 379-389. 